\title{
Two-Dimensional Ising Model with Competing Interactions as a Model for Interacting $\pi$-Rings
}

\author{
A. O'Hare ${ }^{a}$, F.V. Kusmartsev ${ }^{a}$ And K.I. Kugel ${ }^{a, b}$ \\ ${ }^{a}$ Department of Physics, Loughborough University \\ Leicestershire, LE11 3TU, UK \\ ${ }^{b}$ Institute for Theoretical and Applied Electrodynamics \\ Russian Academy of Sciences \\ Izhorskaya Str. 13, Moscow, 125412 Russia
}

\begin{abstract}
We investigate the Ising model on a square lattice with antiferromagnetic exchange between nearest and next-nearest neighbors and show that at low temperatures stripe-like and droplet-like superstructures appear. We show that the competing interactions introduce a strong frustration that could plausibly describe the systems with dipole-dipole type interactions and pay particular attention to arrays of interacting Josephson $\pi$-rings.
\end{abstract}

PACS numbers: 05.50.+q, 75.10.Hk, 74.81.Fa, 03.67.Lx

\section{Introduction}

It is well known that the interplay between the competing interactions in many magnetic and superconducting materials as well as in complex systems leads to a rich phase diagram with a large number of phases and non-trivial types of ordering. As a vivid example of such systems, we investigate here the two-dimensional (2D) Ising model with competing antiferromagnetic nearest-neighbor $(J)$ and diagonal $\left(J^{\prime}\right)$ interactions on a square lattice. It is now well accepted that such a model exhibits two phases with magnetic long-range order: a semiclassical Néel-like magnetic order at small $J^{\prime}$ and a antiferromagnetically coupled ferromagnetic chains (vertical or horizontal stripes) at large $J^{\prime}$. These two ordered phases are separated by an intermediate quantum paramagnetic phase without the long-range order, the nature of which is still under discussion.

An example of a system that may be described by such a model is a chain or a planar array of electrically isolated Josephson $\pi$-rings which could be treated as a set of magnetic moments oriented perpendicular to the plane (i.e. as the Ising spins) and interacting via magnetic dipole forces (taking care to include next-nearest neighbor interactions) [1].

Here, we show that this model exhibits an additional low temperature disordered phase due to the proximity of the lowest energy excited states to the ground state (see also [2]).

\section{Model and a 5-site plaquette}

The Hamiltonian for the Ising model with nearest neighbor and next-nearest (diagonal) neighbor interactions is given by

$$
H=J \sum_{\langle\mathrm{nn}\rangle} s_{i} s_{j}+J^{\prime} \sum_{\langle\mathrm{dn}\rangle} s_{i} s_{j}+h \sum_{i} s_{i},
$$

where $J, J^{\prime}>0, s$ is a two-value Ising variable $s= \pm 1$, $\langle\mathrm{nn}\rangle$ and $\langle\mathrm{dn}\rangle$ denote the summation over sites $i$ and $j$ being respectively nearest neighbors and diagonal neighbors.

For a square lattice with fixed lattice spacing, the ratio $r_{\mathrm{nn}} / r_{\mathrm{dn}}=\sqrt{2}$, since dipole-dipole interactions decay as $\approx 1 / r^{3}$ we have $J / J^{\prime}=2 \sqrt{2}$ for the square lattice. For the sake of generality, we will consider various values of $J / J^{\prime}$ which may be applicable to distorted square lattices.

The simplest system, which may already have non-trivial features but contains all the physics of the model investigated here, is a cluster consisting of five sites. Such a cluster has a similar geometry to the square lattice, i.e. it is a site with four neighbors (a plaquette). Furthermore, we choose this plaquette for the further analysis because it also retains the symmetry and the characteristic geometry of the lattice as a whole (the number of nearest-neighbor and diagonal bonds are equal, see Fig. 1).

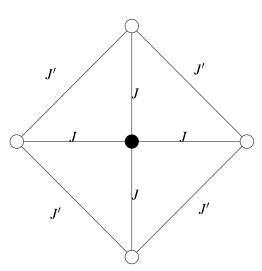

Fig. 1. Square plaquette of the minimum size (with equal numbers of nearest- and next-nearest neighbor bonds). 
The possible configurations of this 5 -site plaquette are easily listed (we have $2^{5}=32$ configurations with different values of energy). Knowing the energy levels and their degeneracy it is possible to write the partition function and calculate the free energy and heat capacity of this plaquette. Plotting the positions of heat capacity maxima in the $T / J^{\prime}-J / J^{\prime}$ plane yields a kind of phase diagram for a small plaquette (Fig. 2). Indeed, the positions of $C\left(T / J^{\prime}, J / J^{\prime}\right)$ peaks could be related to the actual phase transitions occurring in infinite systems. Let us note that at $J / J^{\prime}<2$, the lowest energy corresponds to a state in which ordering occurs via chains of spins with the same direction, whereas at $J / J^{\prime}>2$, the usual two-sublattice antiferromagnetism is the most favorable. However, at non-zero temperatures, there is no boundary between these two phases. Instead, we have a whole quadrant in the $T / J^{\prime}-J / J^{\prime}$ plane (with the vertex at point $(0,2))$, where one could expect numerous phases in the infinite lattice, intermediate between the two-sublattice and chain-like structures. The situation is very much like that of the ANNNI model [3], where the "devil's staircase" of different phases arises at finite temperatures between two main phases existing at zero temperature. Region 3 within this quadrant is bounded by a curve corresponding to the positions of an additional (high-temperature) peak in the temperature dependence of the heat capacity. Therefore, this is another indication that in the infinite system, one should expect in this range a phase with a certain kind of ordering, maybe quite unusual, rather than simply a disordered paramagnetic phase.

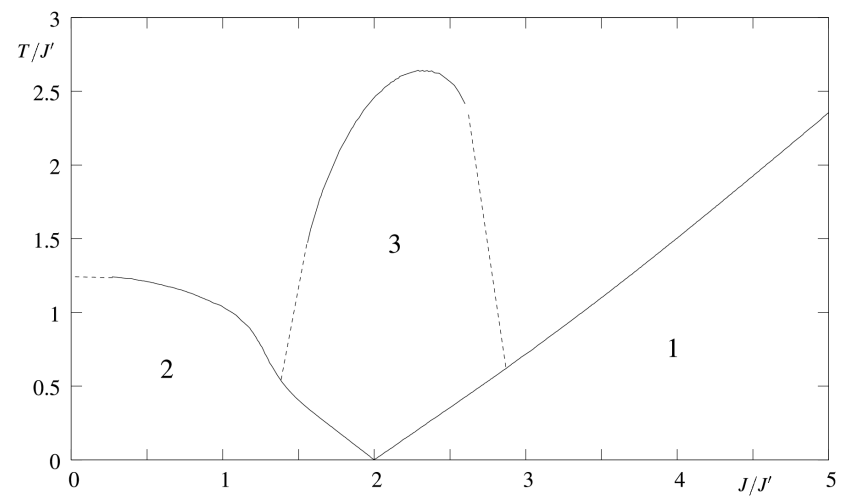

Fig. 2. Phase diagram (positions of the heat capacity peaks) for a 5 -site plaquette. Region 1 corresponds to antiferromagnetic ordering, region 2 corresponds to stripe ordering, while in region 3 , one could expect for the corresponding infinite square lattice multiple phases (similar to those in the ANNNI model) or a phase with a complicated spin ordering.

For the plaquette cluster, the antiferromagnetic ground state, having the zero entropy (non-degenerate), is very difficult to reach. So the system is practically always disordered due to a proliferation of defects. However, the broad peak in specific heat indicates that a true phase transition may arise for large systems.

\section{Larger plaquettes and a glassy state}

Qualitatively, the situation remains the same as we increase lattice size. We calculated the specific heat for an $8 \times 8$ lattice via the transfer matrix method and a $16 \times 16$ lattice via the Monte Carlo method.

The phase diagram obtained for the $8 \times 8$ lattice is plotted in Fig. 3. The comparison of the phase diagrams for $8 \times 8$ and $16 \times 16$ lattices is illustrated in Fig. 4 . We find that at low temperatures there are many energy levels lying close to the ground state effectively trapping the system in one of these locally (meta-)stable states associated with local energy minima separated by large energy barriers.

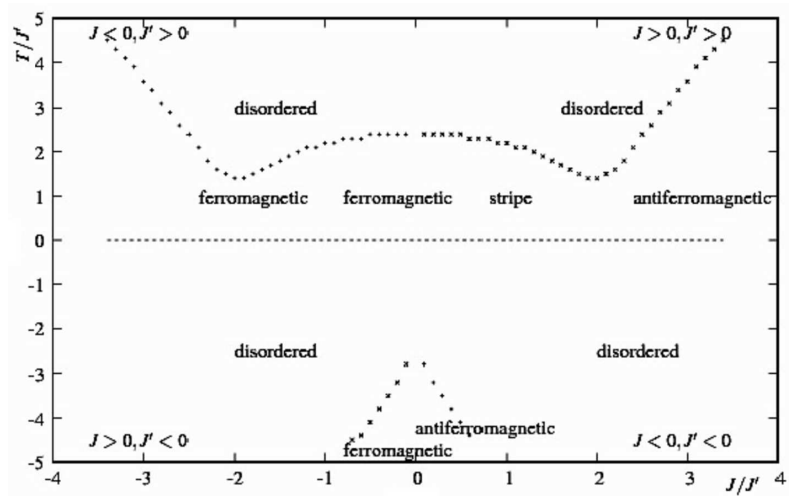

Fig. 3. Phase diagrams for an $8 \times 8$ lattice calculated by the transfer matrix method.

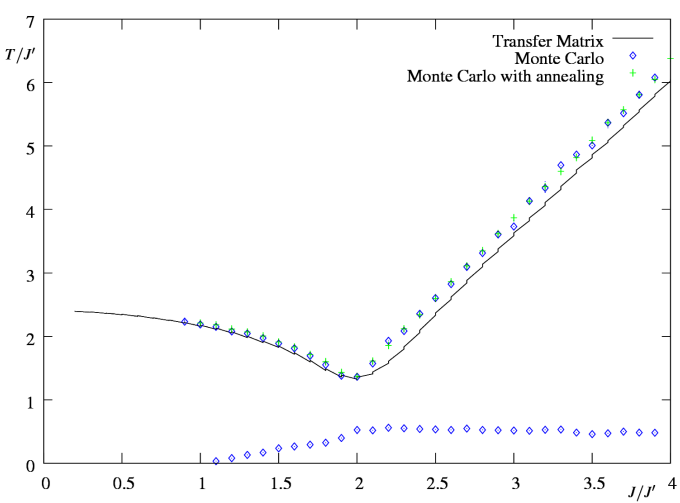

Fig. 4. Peaks in the specific heat of the 2D Ising model with competing interactions on a square lattice using a $16 \times 16$ Monte Carlo simulation and an $8 \times 8$ transfer matrix. The low temperature "phase transition" is not a peak in the specific heat but rather a crossover transition.

At low temperature, one would expect the system to exist in an ordered state with long-range order. However, the appearance of defects (locally stable droplets) 
reduce correlations to a few spins, but temporal correlations due to freezing of the spins can be very strong. Local squared magnetization is given by the average of the autocorrelation functions. This is the order parameter proposed by Edwards and Anderson [4]: $q_{\mathrm{EA}}=$ $\lim _{t \rightarrow \infty} \lim _{V \rightarrow \infty}\left[\left\langle s_{i}\left(t_{0}\right) s_{i}\left(t_{0}+t\right)\right\rangle\right]$.

Spin-glass ordering, if it can be described within the framework of equilibrium statistical mechanics, corresponds to a non-zero value of the Edwards-Anderson order parameter. We can see from Fig. 5 that Monte Carlo simulations clearly show the Edwards-Anderson order parameter exploding as $T / J^{\prime} \rightarrow 0$. This is indicative of spin-glass ordering.

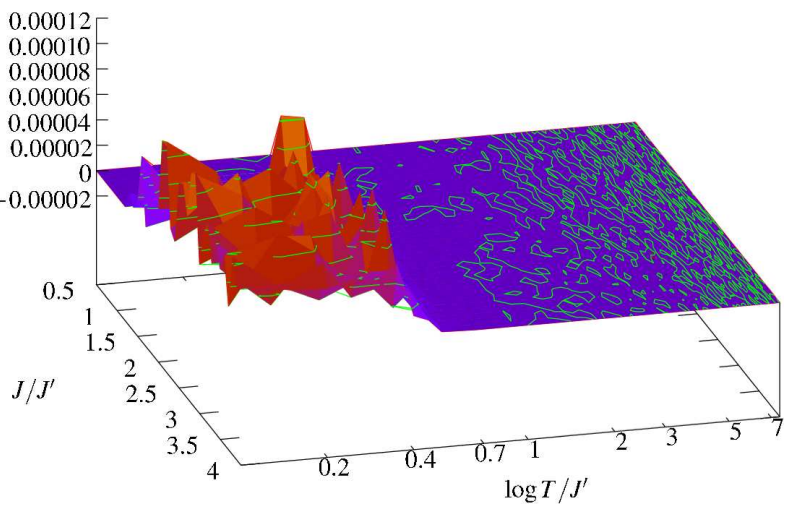

Fig. 5. Edwards-Anderson order parameter for the 2D Ising model with next-nearest neighbor interactions on an $16 \times 16$ square lattice. The explosion of this order parameter at low temperature is an indication of the formation of a spin-glass state.

The low-temperature superstructures that appear in this model are characterized by the formation of locally stable droplets in the background order. This low temperature phase is akin to spin-glass ordering as shown by the Edwards-Anderson order parameter though other order parameters may exist.

\section{Conclusion}

The zero value of entropy at low temperatures shows that the ground state at low temperatures is ordered either as stripes, $J / J^{\prime}<2$ or as the Néel antiferromagnet,
$J / J^{\prime}>2$. However, the recent findings in the framework of this model [2] clearly demonstrate that the energy of defects and dislocations in the lattice is close to the ground state of the system. Therefore, many locally stable (or metastable) states associated with local energy minima separated by energy barriers may appear forming a glass-like state.

Careful preparation of the lattice could result in the system being trapped in this glass-like state which may be used as an initial state for adiabatic quantum computing [2]. Recently, a scalable design that may be used in such a way [5] has been proposed and realized though the range of problems that may be solved may be limited. One such application is a Travelling Salesman Problem [6], which can be represented in the form of more complicated Ising model, with a set of a coupling constants [7].

\section{Acknowledgments}

The work was supported by the ESF network-program AQDJJ, European project CoMePhS, ISTC (grant G1335), and RFBR (project 08-02-00212).

\section{References}

[1] J.R. Kirtley, C.C. Tsuei, Ariando, H.J.H. Smilde, H. Hilgenkamp, Phys. Rev. B 72, 214521 (2005).

[2] A. O'Hare, F.V. Kusmartsev, K.I. Kugel, M.S. Laad, Phys. Rev. B 76, 064528 (2007).

[3] W. Selke, Phys. Rep. 170, 212 (1988).

[4] S.F. Edwards, P.W. Anderson, J. Phys. F 5, 965 (1975).

[5] V. Zakosarenko, N. Bondarenko, S.H.W. van der Ploeg, A. Izmalkov, S. Linzen, J. Kunert, M. Grajcar, E. Il'ichev, H.-G. Meyer, Appl. Phys. Lett. 90, 022501 (2007).

[6] Tien D. Kieu, quant-ph/0601151 (2006).

[7] R. Martoňàk, G.E. Santoro, E. Tosatti, Phys. Rev. E 70, 057701 (2004). 